\title{
Graphene as plasma-compatible blocking layer material for area-selective atomic layer deposition: A feasibility study for III-nitrides
}

Petro Deminskyi, Ali Haider, Evgeniya Kovalska, and Necmi Biyikli

Citation: Journal of Vacuum Science \& Technology A 36, 01A107 (2018); doi: 10.1116/1.5003421

View online: https://doi.org/10.1116/1.5003421

View Table of Contents: https://avs.scitation.org/toc/jva/36/1

Published by the American Vacuum Society

\section{ARTICLES YOU MAY BE INTERESTED IN}

Review Article: Catalysts design and synthesis via selective atomic layer deposition Journal of Vacuum Science \& Technology A 36, 010801 (2018); https://doi.org/10.1116/1.5000587

Thermal adsorption-enhanced atomic layer etching of $\mathrm{Si}_{3} \mathrm{~N}_{4}$ Journal of Vacuum Science \& Technology A 36, 01B104 (2018); https://doi.org/10.1116/1.5003271

Nucleation mechanism during $\mathrm{WS}_{2}$ plasma enhanced atomic layer deposition on amorphous $\mathrm{Al}_{2} \mathrm{O}_{3}$ and sapphire substrates

Journal of Vacuum Science \& Technology A 36, 01 A105 (2018); https://doi.org/10.1116/1.5003361

Influence of $\mathrm{N}_{2} / \mathrm{H}_{2}$ and $\mathrm{N}_{2}$ plasma on binary III-nitride films prepared by hollow-cathode plasma-assisted atomic layer deposition Journal of Vacuum Science \& Technology A 36, 01A110 (2018); https://doi.org/10.1116/1.4998920

Thermal atomic layer deposition of tungsten carbide films from $\mathrm{WCl}_{6}$ and $\mathrm{AlMe}_{3}$ Journal of Vacuum Science \& Technology A 36, 01 A104 (2018); https://doi.org/10.1116/1.5002667

Overview of atomic layer etching in the semiconductor industry Journal of Vacuum Science \& Technology A 33, 020802 (2015); https://doi.org/10.1116/1.4913379

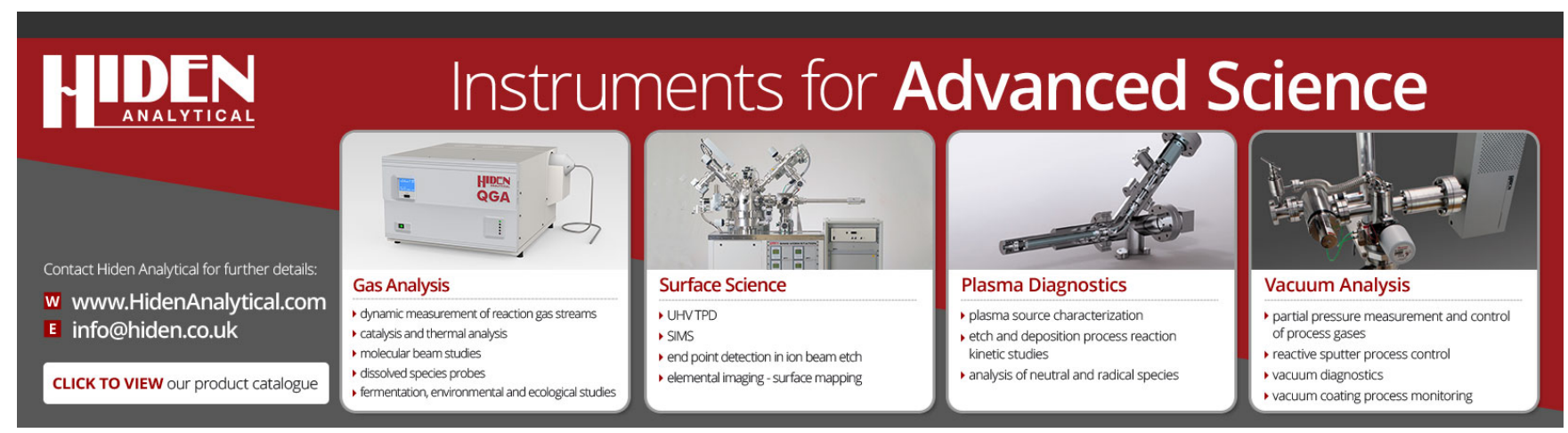




\title{
Graphene as plasma-compatible blocking layer material for area-selective atomic layer deposition: A feasibility study for III-nitrides
}

\author{
Petro Deminskyi \\ National Nanotechnology Research Center, Bilkent University, Bilkent, Ankara 06800, Turkey and \\ Technological and Analytical Research Department, NASU-Institute of Microdevices, Kiev 04136, Ukraine \\ Ali Haider \\ National Nanotechnology Research Center, Bilkent University, Bilkent, Ankara 06800, Turkey and Institute \\ of Materials Science and Nanotechnology, Bilkent University, Bilkent, Ankara 06800, Turkey \\ Evgeniya Kovalska \\ Department of Physics, Bilkent University, Bilkent, Ankara 06800, Turkey \\ Necmi Biyiklia) \\ Department of Electrical and Computer Engineering, University of Connecticut, 371 Fairfield Way, Storrs, \\ Connecticut 06269
}

(Received 5 September 2017; accepted 27 October 2017; published 16 November 2017)

Plasma-assisted atomic layer deposition (PA-ALD) is a promising method for low-temperature growth of III-nitride materials. However, selective film deposition using PA-ALD is challenging mainly due to the plasma-incompatibility of conventional deactivation/blocking layers including self-assembled monolayers and polymers. The main motivation behind this work is to explore alternative plasmaresistant blocking layer materials. Toward this goal, single/multilayered graphene (SLG/MLG) sheets were investigated as potential growth-blocking layers for III-nitride grown via PA-ALD. Prior to PAALD growth experiments, partially graphene-covered $\mathrm{Si}(100)$ samples were exposed to $\mathrm{N}_{2} / \mathrm{H}_{2}$ and $\mathrm{N}_{2}-$ only plasma cycles to evaluate the plasma resistance of SLG and MLG. While SLG degraded fairly quickly showing signs of completely etched areas and rough surface morphology, MLG surface displayed certain degree of plasma-resistance. Based on this result, III-nitride PA-ALD experiments were carried out on MLG-patterned $\mathrm{Si}(100)$ samples. Crystalline III-nitride film deposition was observed on both $\mathrm{Si}(100)$ and graphene surfaces, confirming the rather ineffective nucleation blocking property of graphene surface against PA-ALD process. However, as graphene layers feature relatively weak van der Waals bonds at the substrate/graphene interface as well as between the multilayer graphene interfaces, conventional lift-off process was sufficient to remove the deposited excessive nitride films. $\mathrm{InN}$ and AlN-coated samples were ultrasonicated, and blocked/unblocked surfaces were characterized using scanning electron microscopy, $\mathrm{x}$-ray photoelectron spectroscopy, and spectroscopic ellipsometer. While $\sim 50 \mathrm{~nm}$ thick films were measured in the open $\mathrm{Si}(100)$ areas, graphene-coated sample portions exhibited limited material growth in the range of 5-15 nm. Although not completely, the MLG surface has considerably blocked the PA-ALD growth process resulting in a usable thickness difference, enabling growth selectivity with postgrowth etch process. An Ar-based physical dry etching recipe was utilized to completely etch the unwanted nitride films from graphene coated area, while about 30 and $40 \mathrm{~nm}$ thick $\mathrm{InN}$ and AlN films remained on the nonblocked parts of the samples, respectively. As a result, selective deposition of PA-ALD grown AIN and InN has been achieved via graphene-assisted lift-off technique along with subsequent dry-etch process, achieving a maximum growth selectivity of $\sim 40 \mathrm{~nm}$. With further process recipe optimization and integrating with a suitable patterning technique, the demonstrated graphene-assisted lift-off technique might offer an alternative feasible pathway toward area-selective deposition of III-nitrides and other plasma-necessitating materials. Published by the AVS. https://doi.org/10.1116/1.5003421

\section{INTRODUCTION}

The ability of depositing specific material(s) only on certain material(s) surfaces has been of significant interest, particularly for the microelectronics industry, as it provides self-aligned bottom-up processing solutions which reduces the burden of costly and complex top-down lithography. A variety of selective deposition techniques have been

$\overline{{ }^{a} \text { Electronic mail: necmi.biyikli@uconn.edu }}$ developed successfully and are being used in high-volume chip manufacturing, including selective cobalt (Co) chemical vapor deposition process on copper $(\mathrm{Cu})$ surfaces which significantly enhances the electromigration resistance of $\mathrm{Cu}$ interconnects. ${ }^{1}$ However, the ever-shrinking device sizes and layer thicknesses, increasingly three-dimensional structural complexity, inclusion of a broader range of materials, and the need for atomic-precision processing mandates the development of selective deposition recipes for a wider selection of materials and surfaces. ${ }^{2}$ Toward this goal, the 
atomic layer deposition (ALD) community initiated efforts to advance selective materials processing more than a decade ago, which more recently became a significant subfield within ALD research, most widely known as area-selective ALD (AS-ALD). ${ }^{3-7}$

Ideal ALD processes are based on self-limiting surface reactions in which the film nucleation critically relies on chemical interactions between gaseous precursor molecules and the solid substrate surface, providing an attractive opportunity for performing selective deposition via chemical surface modifications. ${ }^{8}$ The rapidly progressing AS-ALD literature concentrates entirely on oxides and metals either via area-deactivated, ${ }^{9-16}$ area-activated, ${ }^{17-20}$ or inherent surface selectivity ${ }^{21,22}$ approaches. Selective deposition recipes for nitride materials are however still missing, mainly due to the need for nitrogen plasma coreactants, which enable the nitrogen incorporation at reduced self-limiting growth temperatures. Such plasma coreactants feature reactive etch species which prove to be harmful for conventional growth blocking/inhibition layer materials used in AS-ALD including self-assembled monolayers and various polymers. ${ }^{23}$ They quickly and aggressively degrade/etch these blocking layers, leaving the inherently selective deposition as the only alternative solution, which is at least as challenging due to the nonselective nucleation tendency of nitrides and barrierless character of plasma.

Recently, two groups challenged the problem of developing selective plasma-assisted ALD (PA-ALD) recipes for oxide compounds: Vallat et al. reported on the inherently selective deposition of $\mathrm{Ta}_{2} \mathrm{O}_{5}$ on TiN surfaces using PAALD featuring oxygen plasma, where they have achieved a viable nucleation delay on the $\mathrm{Si}$ surface. ${ }^{24}$ Combining with periodic etching cycles to remove the unwanted nucleation on $\mathrm{Si}$, they were able to obtain selective $\mathrm{Ta}_{2} \mathrm{O}_{5}$ deposition on TiN surface only. The second recent attempt was carried out by Mameli et al., similarly utilizing oxygen plasma for the PA-ALD of $\mathrm{SiO}_{2} \cdot{ }^{25}$ Deposition selectivity, however, was achieved using a completely different approach, where a three-step (ABC-type) ALD reaction was developed featuring an inhibitor molecule exposure step which adsorbs selectively on the no-growth-targeted surface. This method overcomes the need for periodic etch cycles and successfully demonstrates selective PA-ALD of $\mathrm{SiO}_{2}$ films on several oxide and nitride surfaces while providing nucleation delays on a few others.

In this work, we attempt to demonstrate the feasibility of attaining selective deposition of III-nitride thin films via PAALD by exploring an unconventional blocking/inhibition layer material which is known for its chemical inertness, mechanical stability, and attractive electronic properties: graphene. Our motivation was mainly threefold: (1) to observe nitrogen plasma-graphene interaction results and determine plasma compatibility of graphene; (2) to evaluate III-nitride PA-ALD inhibition properties of graphene surface for possible selective III-nitride deposition; (3) to facilitate the inherent van der Waals forces with graphene-assisted lift-off for III-nitride patterning. Our feasibility study demonstrates that multilayered graphene (MLG) exhibits sufficient plasma resistance and can be used as a blocking layer for InN and AIN PA-ALD growth, resulting in a maximum growth selectivity of $\sim 40 \mathrm{~nm}$ after a combined lift-off and dry-etch process.

\section{EXPERIMENT}

Figure 1 summarizes the experimental methodology utilized in our feasibility study: (1) CVD-grown single-layer graphene (SLG) and MLG sheets are transferred onto cleaved $\mathrm{Si}(100)$ substrates so that arbitrarily patterned graphene/Si samples are formed. At this stage, plasma resistance of the graphene layers were checked with plasma-only exposure cycles. (2) Low-temperature PA-ALD growth of III-nitride thin films onto the partially graphene coated samples, after which the film thicknesses on graphene and Si surfaces were checked to evaluate any nucleation/growth delay on graphene area. (3) Ultrasonication (US) of the III-nitride coated samples to facilitate the removal of III-nitride films on graphene via lift-off process. (4) The resulting sample is checked for any growth selectivity by measuring film thickness in open $\mathrm{Si}(100)$ and graphene-coated areas. Post-lift-off dry etching is utilized to remove any deposited films in the graphene-protected sample parts.

\section{A. Material growth and processing}

The high-temperature CVD-based SLG and MLG synthesis on copper and nickel substrates, respectively, and the polymer-assisted transfer procedure can be summarized as the following. ${ }^{26,27}$ (1) SLG synthesis and transfer: CVDbased SLG synthesis was carried out inside a quartz chamber on a $25 \mu \mathrm{m}$ thick copper foil (Alfa Aesar). The copper foil was annealed at $1035^{\circ} \mathrm{C}$ under vacuum in $80 \mathrm{sccm} \mathrm{H}_{2}$ flow (a)

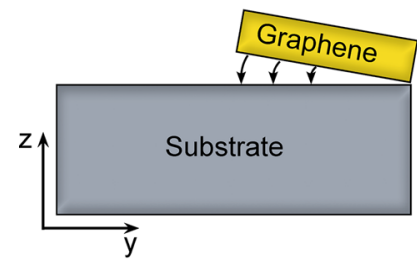

(c)

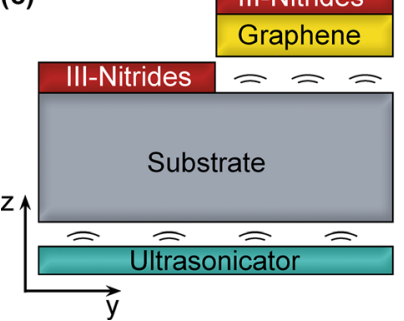

(b)

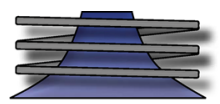

III-Nitrides

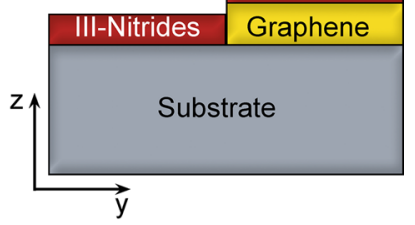

(d)

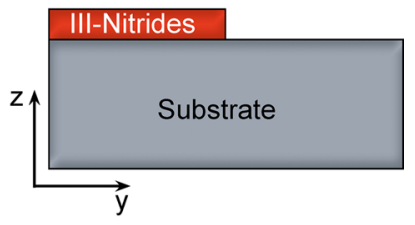

FIG. 1. (Color online) Schematic illustration of the process flow utilized to evaluate the selective deposition of III-nitride materials using graphene blocking layers. (a) CVD-grown graphene transfer on Si substrate; (b) IIInitride growth via PA-ALD; (c) ultrasonic lift-off process of III-nitride films grown on graphene layers; (d) resulting patterned III-nitride film after liftoff process along with additional dry etching used to remove any deposition underneath the graphene layers. 
for $30 \mathrm{~min}$, and graphene was synthesized with the additional $40 \mathrm{sccm}$ flow of $\mathrm{CH}_{4}$ during the subsequent $30 \mathrm{~min}$. The graphene on copper was unloaded from the CVD-chamber when the substrate temperature decreased below $60^{\circ} \mathrm{C}$. A photoresist S1813 (Shipley Company) supported transfer method was utilized to transfer the graphene layer to the $\mathrm{Si}$ wafer surface. $\mathrm{FeCl}_{3}$ and acetone-assisted etching process were performed in order to remove $\mathrm{Cu}$-foil and photoresist, respectively. (2) MLG synthesis and transfer: MLG samples were synthesized on a $25 \mu \mathrm{m}$ thick nickel foil (Alfa Aesar) by $\mathrm{CVD}$ at $900{ }^{\circ} \mathrm{C}$ under ambient pressure. $\mathrm{H}_{2}, \mathrm{Ar}$, and $\mathrm{CH}_{4}$ gases were used during the growth process. Flow rates of the $\mathrm{CH}_{4}, \mathrm{H}_{2}$, and Ar were set as 30,100, and $100 \mathrm{sccm}$, respectively. Growth time was $5 \mathrm{~min}$ in order to obtain $\sim 10$ layers of graphene. After terminating the growth, the samples were left for fast cooling to room temperature. Subsequently, MLG/Ni sample is soaked into $1 \mathrm{M} \mathrm{FeCl}_{3}$ solution in order to separate the MLG from Ni substrate. MLG was then dipped into deionized (DI) water and transferred onto $\mathrm{Si}$ wafer via fishing method. Finally, samples were placed into oven for $15-20 \mathrm{~min}$ for drying at $75^{\circ} \mathrm{C}$. To improve contact between the surface and graphene sheets, isopropanol and acetone rinsing were utilized.

Plasma resistance and durability measurements, as well as the subsequent III-nitride deposition experiments, were carried out in a modified Fiji F200-LL remote-plasma ALD reactor (Veeco/CambridgeNanoTech, Inc.), which is backed by an Adixen ACP 120G dry scroll vacuum pump. The original system was customized by replacing the quartz-based inductively coupled plasma (ICP) source with a stainless steel hollow-cathode plasma (HCP) source (Meaglow, Ltd., Thunder Bay, ON, Canada). Brief reasoning behind the plasma source choice is the dramatic reduction observed in oxygen impurity levels within the nitride films after the replacement. Detailed experimental results before and after this modification can be found elsewhere. ${ }^{28,37}$ The original RF power supply (Seren IPS, Inc., R301), matching network controller (Seren IPS, Inc., MC2), and automatic matching network (Seren IPS, Inc., AT-3) units were used to activate the HCP discharge. Substrates were kept at deposition temperature for at least $20 \mathrm{~min}$ before the growth process was started. The rotation speed of the Adixen ATH 400M turbo pump was adjusted in order to keep the reactor pressure fixed at $\sim 150$ mTorr during growth sessions, whereas the base pressure of the system was lower than $10^{-5}$ Torr. Prior to graphene transfer, $\mathrm{Si}(100)$ samples were cleaned by sequential ultrasonic agitation in 2-propanol, acetone, and methanol, followed by rinsing with DI water and drying with $\mathrm{N}_{2}$, and were then loaded into the PA-ALD reactor.

Compatibility and durability of SLG and MLG layers against $\mathrm{N}_{2}$-only and $\mathrm{N}_{2} / \mathrm{H}_{2}$ plasma was tested under $50 \mathrm{sccm}$ and $50 / 50 \mathrm{sccm}$ gas flow rates for $\mathrm{N}_{2}$-only and $\mathrm{N}_{2} / \mathrm{H}_{2}$ plasmas, under $300 \mathrm{~W}$ plasma power. Ar is used as the carrier gas for both $\mathrm{N}_{2}$-only and $\mathrm{N}_{2} / \mathrm{H}_{2}$ plasma; therefore, the plasma is a mixture of $\mathrm{N}_{2} / \mathrm{Ar}$ and $\mathrm{N}_{2} / \mathrm{H}_{2} / \mathrm{Ar}$ plasma, including nitrogen and hydrogen radicals, Ar neutrals, and possibly $\mathrm{Ar}+$ ions. $\mathrm{N}_{2}, \mathrm{H}_{2}$, and Ar gases were of $99.998 \%$ purity, which were further purified via in-line Microtorr (MCL-
702F) gas purifier units. The remote RF-plasma (300 W) was activated each cycle for $40 \mathrm{~s}$. Unless stated otherwise, the system was purged for $10 \mathrm{~s}$ after each plasma exposure. Experiments were carried out for 50 and 100 cycles, whereafter the single-layer and multilayered graphene sample surfaces were characterized. All growth experiments were carried out at a fixed substrate temperature of $200^{\circ} \mathrm{C}$.

For III-nitride PA-ALD, the unit growth cycle for AlN was formed by trimethylaluminum pulse $(0.06 \mathrm{~s})$, Ar purge $(10 \mathrm{~s}), \mathrm{N}_{2} / \mathrm{H}_{2}$ plasma exposure (40 s), and Ar purge (10 s). For InN, on the other hand, unit growth cycle consisted of trimethylindium pulse $(0.07 \mathrm{~s})$, Ar purge $(10 \mathrm{~s}), \mathrm{N}_{2}$-only plasma exposure (40 s), and Ar purge (10s). It should be noted that, while our optimized $\mathrm{InN}$ deposition recipe required a $\mathrm{N}_{2}$-plasma exposure duration of $100 \mathrm{~s}$ to maximally complete the ligand-exchange reactions, in order to minimize the plasma-damage on graphene blocking layers, we have limited the $\mathrm{N}_{2}$-plasma duration to $40 \mathrm{~s}$ (same as $\mathrm{N}_{2}$ / $\mathrm{H}_{2}$ plasma exposure used for AlN) during our experiments. Detailed self-limiting growth characteristics for PA-ALDgrown AlN, GaN, and InN can be found elsewhere. ${ }^{28-30}$

Ultrasoniction for the lift-off process was carried out in a manual Bransonic MH 1.91 ultrasonic cleaner (BransonEmerson) at room temperature for 30-60 s. The final dry Aretch process was accomplished using a 4-in. wafer compatible ICP etch reactor (SPTS Technologies, ICP-F 615-LL) with an $\mathrm{Ar}$ flow of $20 \mathrm{sccm}$, while the coil and platen power were maintained as 450 and $100 \mathrm{~W}$, respectively.

\section{B. Materials characterization}

Raman spectroscopy was used to characterize the graphene layers (SLG and MLG) before and after plasma exposure cycles via a scanning near-field optical microscope system featuring a built-in laser at $514 \mathrm{~nm}$ (Witec, Alpha300 RS). In order to measure the thickness of the deposited AlN and InN films, a variable-angle spectroscopic ellipsometer (V-VASE, J.A. Woollam Co., Inc., Lincoln, NE) is utilized, coupled with a rotating analyzer and xenon light source. The ellipsometric spectra were collected at three angles of incidence $\left(65^{\circ}, 70^{\circ}\right.$, and $\left.75^{\circ}\right)$ to yield adequate sensitivity over the full spectral range. Film thickness values were extracted by fitting the spectroscopic ellipsometer data using Cauchy model, while the substrate was taken as default $\mathrm{Si}(100)$ in VVase Woollam software. Elemental composition and chemical bonding states of the AlN/Si, AlN/SLG, AlN/MLG, InN/ $\mathrm{Si}, \mathrm{InN} / \mathrm{SLG}$, and InN/MLG samples/surfaces before and after lift-off were obtained by x-ray photoelectron spectroscopy (XPS) measurements using Thermo Scientific KAlpha spectrometer (Thermo Fisher Scientific, Waltham, MA) with a monochromatized Al $\mathrm{K} \alpha$ x-ray source (spot size $\sim 400 \mu \mathrm{m}$ ). All peaks in XPS survey scans are referenced to $\mathrm{C}$ 1s peak for charge correction, and quantification of survey scans has been performed using Avantage software. Surface morphologies of the bare, plasma-exposed, III-nitride coated, and lift-off processed graphene/Si(100) surfaces were revealed using a scanning electron microscope (SEM) system (Nova NanoSEM 450, FEI, Hillsboro, OR). The 

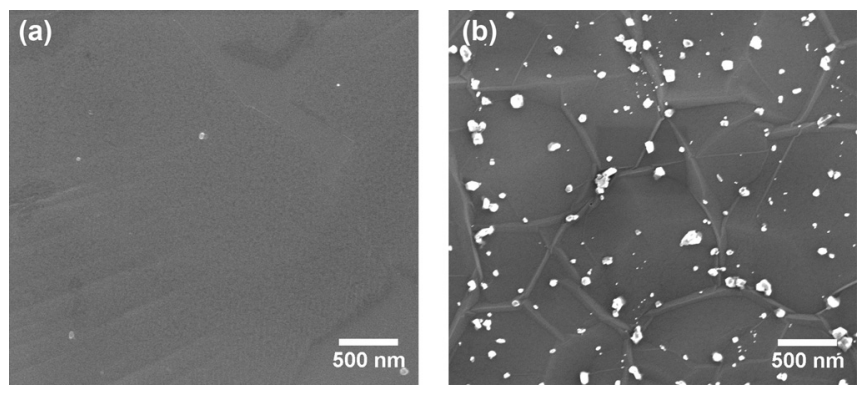

FIG. 2. SEM images of (a) single layer graphene synthesized at $1035^{\circ} \mathrm{C}$ and (b) multilayer graphene synthesized at $850^{\circ} \mathrm{C}$.

detailed structural properties including XRD and TEM analysis of both PA-ALD grown AlN and InN films were previously reported elsewhere. ${ }^{28,30}$

\section{RESULTS AND DISCUSSION}

\section{A. Plasma resistance of graphene layers}

As first step in our study, we evaluated the influence of $\mathrm{N}_{2}$-only and $\mathrm{N}_{2} / \mathrm{H}_{2}$ plasma on SLG and MLG layers as we utilize these plasma chemistries during the PA-ALD growth of AlN, GaN $\left(\mathrm{N}_{2} / \mathrm{H}_{2}\right)$, and $\mathrm{InN}\left(\mathrm{N}_{2}\right.$-only) films. To eliminate any possible negative impact of graphene transfer process (mechanical damage, cracks/defects, nonuniform stiction, etc.), the $\mathrm{N}_{2}$-only and $\mathrm{N}_{2} / \mathrm{H}_{2}$ plasma resistance study was carried out on $\mathrm{Cu} / \mathrm{Ni}$ foil substrates (directly after CVD growth) before they were transferred onto $\mathrm{Si}(100)$ substrate samples. SLG/Cu-foil and MLG/Ni-foil surface SEM images are shown in Figs. 2(a) and 2(b), respectively. It is apparent that the SLG/Cu sample features considerably lower amount of surface contamination when compared to MLG/Ni sample. The random-sized and shaped white spots on the MLG flake-surface correspond to residual metallic $\mathrm{Ni}$ nanoparticles developed during the MLG CVD-growth process, which was confirmed by XPS analysis, revealing the Ni $2 p$ peak around $853 \mathrm{eV}$.

The presence and quality of SLG and MLG were confirmed via Raman spectroscopy as shown in Figs. 3(b) and $3(d)$, which reveals the presence of the peaks related to Gband and 2D-band of graphene around 1585 and $2700 \mathrm{~cm}^{-1}$, respectively, correlating well with literature results. ${ }^{31-33}$ The MLG/Ni sample shows typical multilayer graphene characteristics with lower-intensity and broader 2D peak, while $\mathrm{SLG} / \mathrm{Cu}$ data suffers from the $\mathrm{Cu}$ background signal which makes it difficult to comment on the $I_{2 \mathrm{D}} / I_{G}$ ratio, expected to be around 2 for ideal single-layer graphene surface.

Figures 3(a) and 3(c) depict the SLG and MLG surface SEM images after 50 cycles of $\mathrm{N}_{2}$-only plasma treatment. When compared to the as-grown sample surface, SLG shows clear signs of significant material degradation and loss of integrity [inset of Fig. 3(a)] resulting in highly rough surface. Based on this result, we did not continue further upto 100cycle plasma testing. This reflects in the Raman signal as well as the $G$ and 2D peak intensities decrease after plasma exposure. On the other hand, MLG surface exhibits lower overall surface damage, most probably due to the
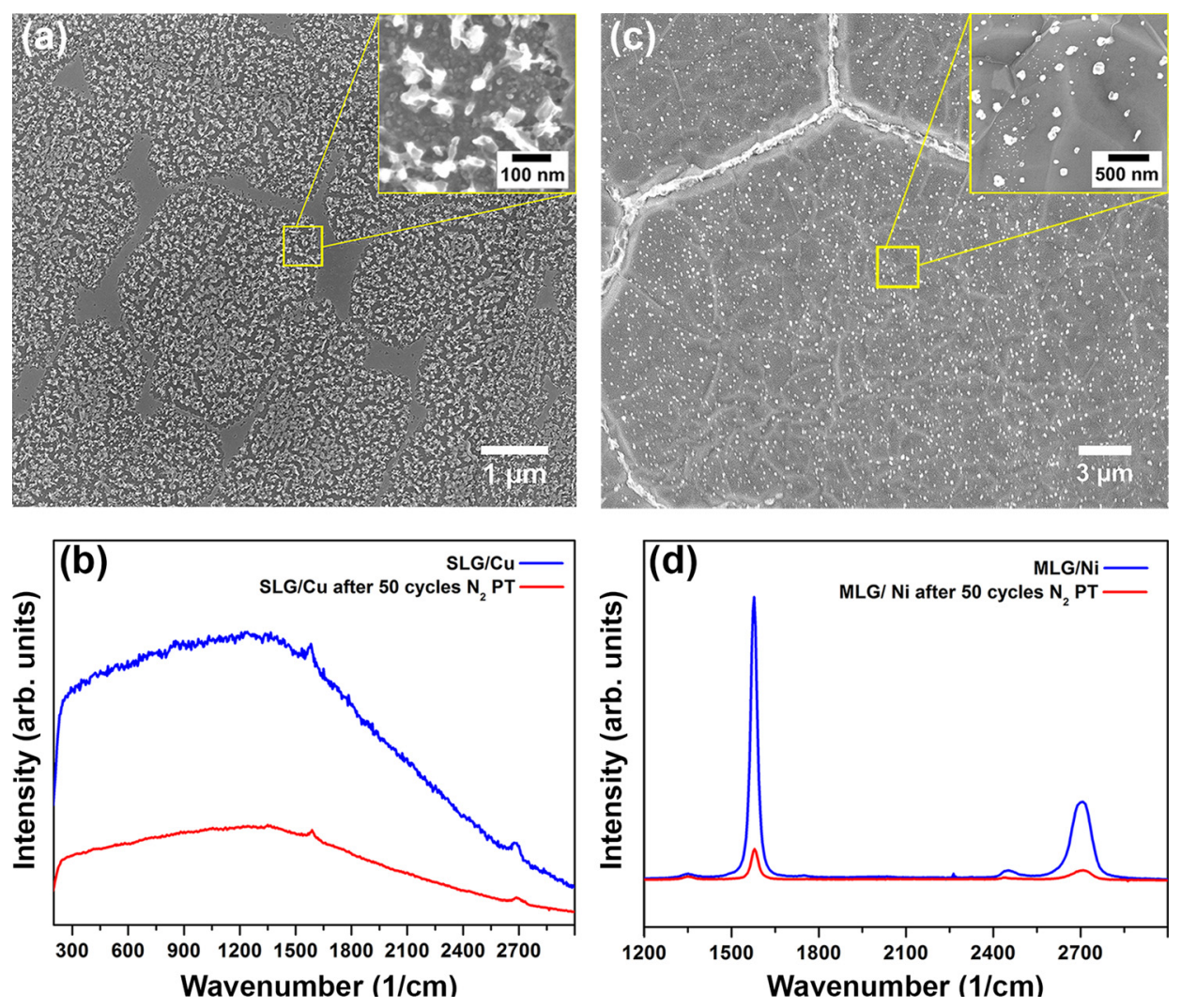

FIG. 3. (Color online) [(a), (c)] SEM images of SLG/Cu-foil and MLG/Ni-foil sample surfaces after 50 cycles of $\mathrm{N}_{2}$ plasma treatment, [(b), (d)] Raman spectroscopy measurements of SLG and MLG samples before and after 50-cycle $\mathrm{N}_{2}$ plasma exposure. 
multilayered structure, i.e., eventhough the upper graphene layers are possibly damaged, underlying layers are still intact and preserve the continuous graphene layers [inset of Fig. 3(c)]. The decreased Raman peaks confirm this hypothesis as the intensities showed considerable decrease while still displaying $G$ and 2D peaks. To gain an insight on the magnitude of plasma-influenced material loss in graphene layers, we carried out XPS measurements on SLG samples exposed to extended $(100 \mathrm{~s}) \mathrm{N}_{2}$-plasma exposures for 50 cycles. When compared to the $40 \mathrm{~s}$ plasma cycles, the resulting carbon content showed a strong decrease from $\sim 19$ to $\sim 3$ at. \%, which confirms the relatively significant material loss due to $\mathrm{N}_{2}$-plasma treatment.

Figure 4 displays the SEM and Raman spectroscopy measurements for the SLG/Cu and MLG/Ni samples after 50 and 100 cycles of $\mathrm{N}_{2} / \mathrm{H}_{2}$ plasma treatment. The SEM images show similar difference between SLG and MLG samples with disintegrated and eroded SLG surface as opposed to the continuous and intact MLG surface structure. When compared with the $\mathrm{N}_{2}$-only plasma treated samples, the pronounced Raman peaks for $\mathrm{N}_{2} / \mathrm{H}_{2}$ plasma treated samples show less degradation: both $\mathrm{G}$ and $2 \mathrm{D}$ peaks for 50-cycle data are higher for $\mathrm{N}_{2} / \mathrm{H}_{2}$ plasma. Possible reasons why $\mathrm{N}_{2} /$ $\mathrm{H}_{2}$ plasma exhibits a softer erosion influence on graphene layers might be partially explained with the reported functionalization and passivation effect of hydrogen plasma which leads to the formation of $\mathrm{C}-\mathrm{H}$ bonds on the graphene surface. ${ }^{34}$ We speculate that such partially functionalized/ passivated graphene surface provides an increased etchresistance against the sputtering ${ }^{35,36}$ mechanism of energetic
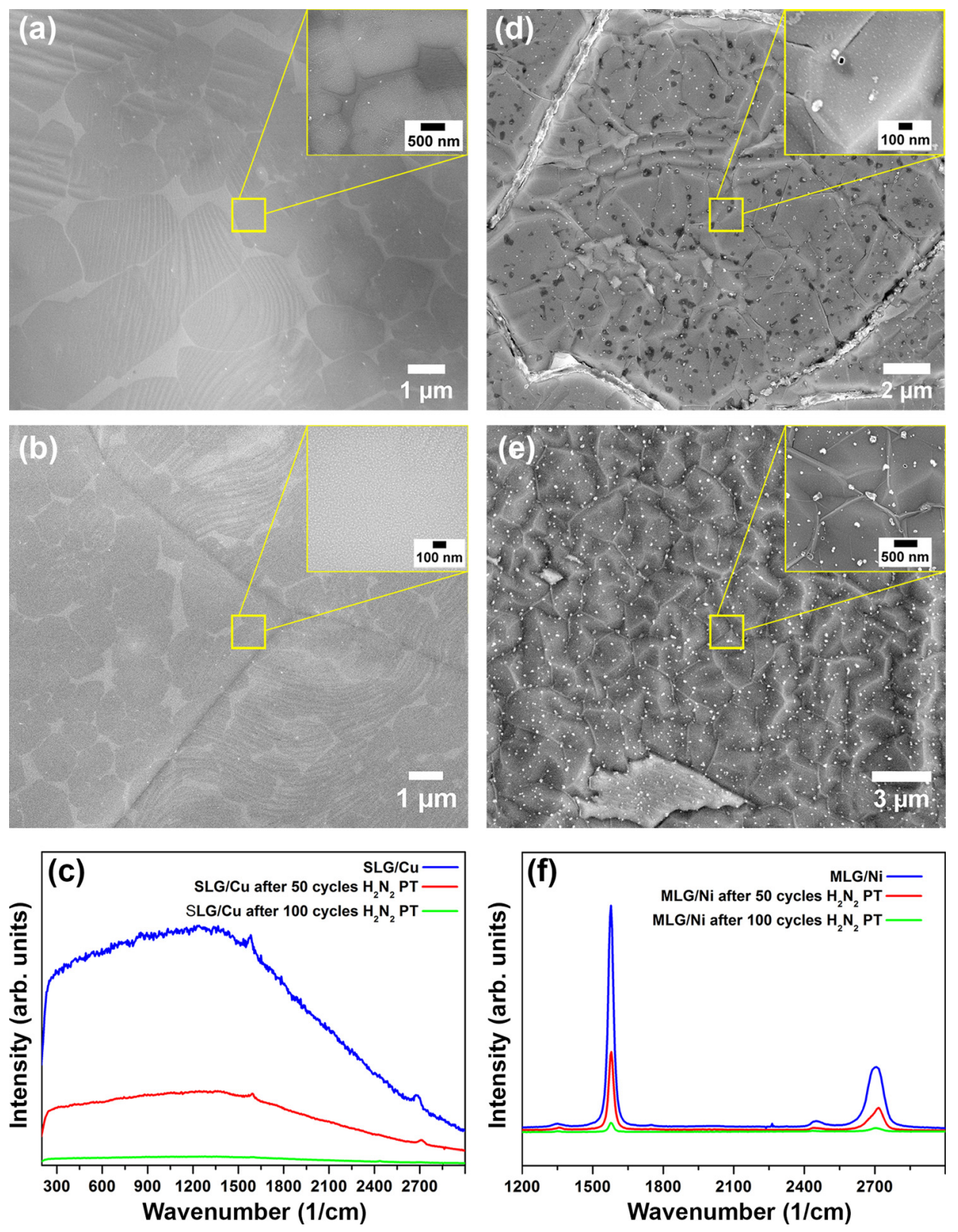

FIG. 4. (Color online) SEM images of SLG/Cu sample after (a) 50 and (b) 100 cycles of $\mathrm{N}_{2} / \mathrm{H}_{2}$ plasma treatment. SEM images of MLG/Ni sample after (d) 50 and (e) 100 cycles of $\mathrm{N}_{2} / \mathrm{H}_{2}$ plasma treatment. Raman spectroscopy measurements of (c) SLG/Cu and (f) MLG/Ni samples before and after 50 and 100 cycles of $\mathrm{N}_{2} / \mathrm{H}_{2}$ plasma treatment. 

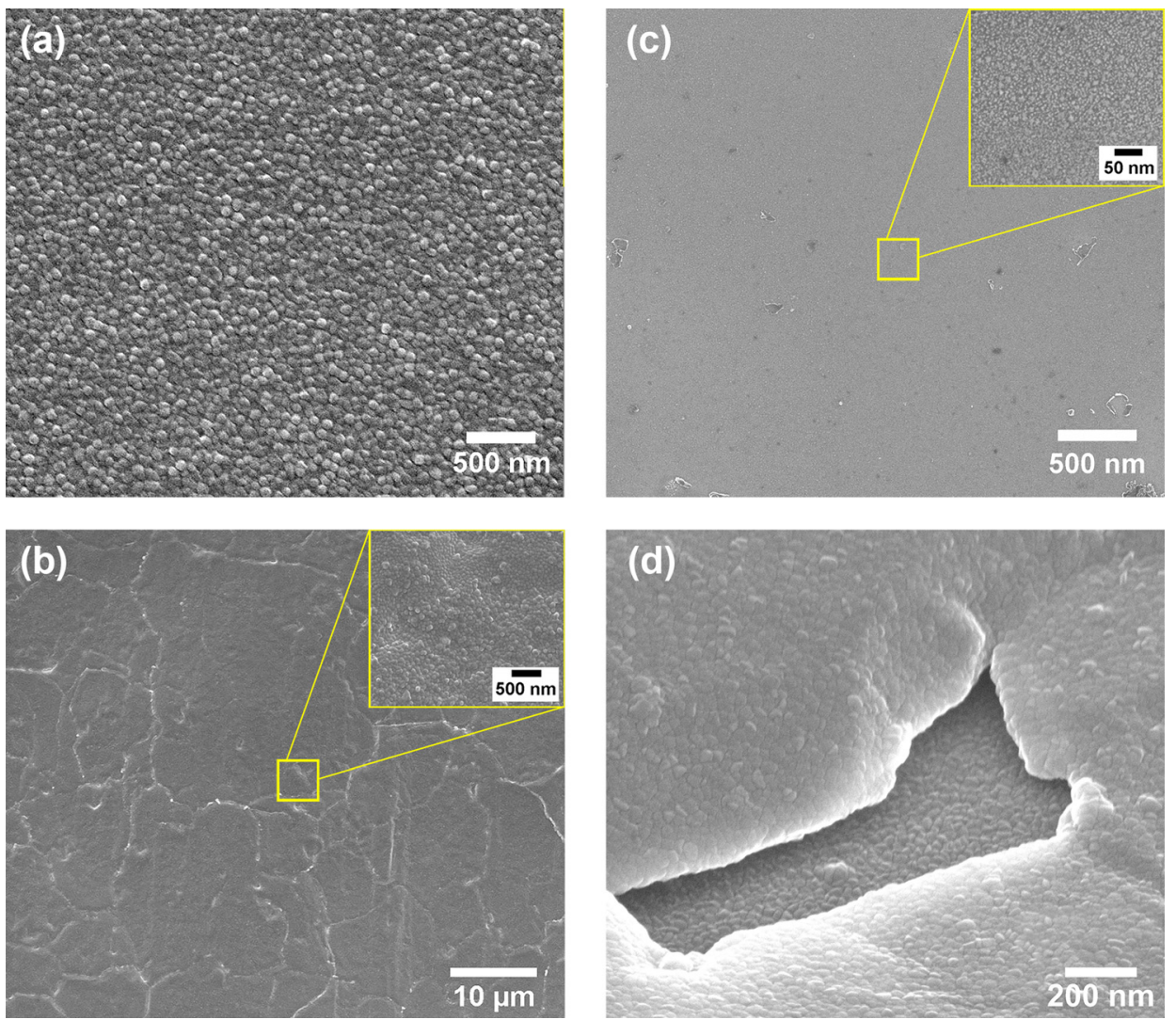

FIG. 5. (Color online) SEM images of (a) InN film on Si(100) surface; (b) InN film on MLG surface; (c) Si surface after lift-off process (this image belongs to the area where MLG blocking layer was present); and (d) InN on partially cracked MLG surface leading to growth on Si as well.

nitrogen radicals. However, this hypothesis needs to be confirmed with further experiments which are currently being planned.

As for the plasma-resistance of SLG and MLG samples, we can conclude that MLG shows a better durability against both $\mathrm{N}_{2}$-only and $\mathrm{N}_{2} / \mathrm{H}_{2}$ plasma exposures by preserving its integrity for at least $50 \mathrm{~N}_{2}$-only and $100 \mathrm{~N}_{2} / \mathrm{H}_{2}$ plasma cycles. Based on this result, MLG qualifies as a potential blocking or lift-off layer for III-nitride PA-ALD processes while SLG would possibly not be able to block III-nitride film growth. Therefore, we decided to proceed with film growth experiments using carefully transferred MLG layers only.

\section{B. III-nitride PA-ALD and graphene-assisted lift-off}

In order to evaluate the PA-ALD growth blocking performance of MLG layers, we have grown InN and AlN films on partially MLG-covered $\mathrm{Si}(100)$ samples. The reasoning behind our choice of InN and AlN lies in the optimized plasma chemistry: while AlN necessitates the usage of $\mathrm{N}_{2} / \mathrm{H}_{2}$ mixture, crystalline $\mathrm{InN}$ films requires $\mathrm{N}_{2}$-only plasma. ${ }^{28-30}$ Such a choice also allows us to compare the MLG blocking performance against the two different plasma environments.

\section{1. $\mathrm{N}_{2}$-only plasma case: Selective InN deposition results}

Figures 5(a) and 5(b) show the SEM images of the open $\mathrm{Si}(100)$ and MLG-covered surfaces, respectively, after 700- cycle InN deposition experiment, which corresponds to a film thickness of $\sim 50 \mathrm{~nm}$ on Si surface (growth-per-cycle of $\sim 0.7 \AA$ ). Both $\mathrm{Si}(100)$ and MLG surface displays uniformly distributed granular deposition of $\mathrm{InN}$ with similar thickness, which proves that graphene surface does not provide any growth blocking/inhibiting property. Although not growth inhibiting, the MLG layer can still be utilized as a lift-off layer, owing to the relatively weak van der Waals (VdW) forces between graphene-Si as well as between the neighbor layers of the MLG stack. To facilitate such a lift-off process, gentle ultrasonication process might provide the necessary force to lift-off the MLG flakes together with the excessive InN film grown on top.

Figure 5(c) depicts the SEM image of the lifted-off $\mathrm{Si}(100)$ surface after ultrasonication process, which still shows certain InN growth in the area where MLG layer was protecting. MLG behaved not as an ideal blocking/barrier layer for InN growth, however, provided partial blockage resulting in a reduced $\mathrm{InN}$ film thickness changing in the range of $5-15 \mathrm{~nm}$. Compared to the $\sim 50 \mathrm{~nm}$ film thickness on unprotected open $\mathrm{Si}(100)$ regions, this should correspond to an effective blocking thickness of more than $\sim 35 \mathrm{~nm}$. Such a difference hints to use a subsequent unselective etching process, by which the unwanted $5-15 \mathrm{~nm} \mathrm{InN}$ film is etched away, leaving $>35 \mathrm{~nm}$ thick InN film on only the non-MLG covered open $\mathrm{Si}(100)$ areas. Before going into the details of post-lift-off etching process results, the uncomplete shielding performance of MLG deserves a brief 
discussion. Among other possible reasons, we believe that mainly the defects and grain boundaries formed during MLG synthesis which might degrade quickly under plasma exposure as well as the unwanted cracks and imperfections at the sides/corners of the flakes developed during the transfer process provide sufficient gaps for the diffusion of organometallic precursors and plasma gases underneath the MLG surface. Moreover, nonperfect VdW stiction might result in additional room for the precursor molecules and plasma radicals to find suitable nucleation sites on the MLG-protected surface. Figure 5(d) shows a high-resolution SEM image of a crack formed in the MLG layer, where the exposed $\mathrm{Si}(100)$ area underneath is exposed to full-capacity ALD growth while the surrounding MLG-covered underneath area would also be vulnerable to unwanted $\mathrm{InN}$ deposition.

Figures 6(a) and 6(b) represent the measured XPS data from unblocked $\mathrm{Si}(100)$ area and MLG-protected area where MLG and the excessive InN film on top of it is lifted-off subsequently and as a function of post-lift-off Ar-etch duration. The In $3 d$ peak on unprotected $\operatorname{Si}(100)$ area does not change with ultrasonication process as expected, while in the MLGcovered area a significant drop in the peak intensity is observed, confirming the lower amount of film growth
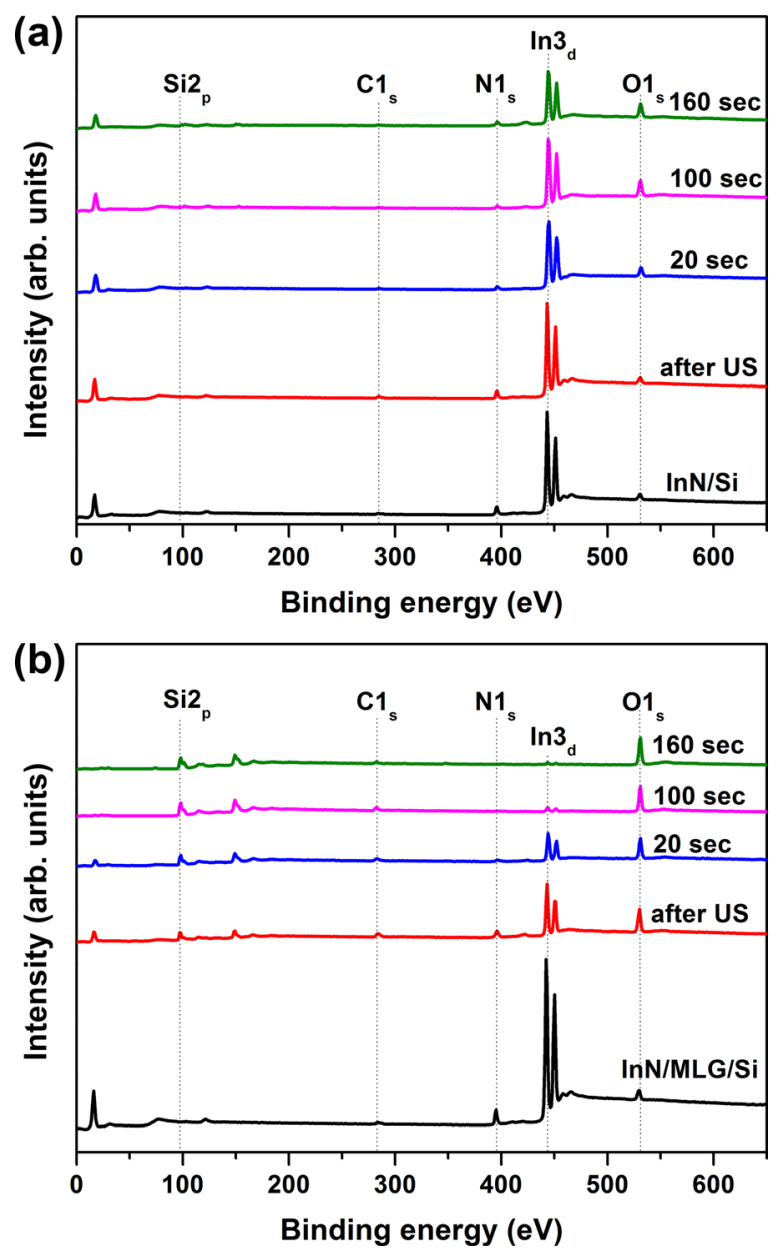

FIG. 6. (Color online) XPS survey scans of (a) InN/Si and (b) InN/MLG/Si surface areas before and after US and as a function of different Ar-etch times. underneath MLG. Subsequent Ar-etch process in the ICP reactor reduces the In peak intensities for both $\operatorname{Si}(100)$ and lifted-off MLG regions: In 3d peak is nearly diminished at $100 \mathrm{~s}$, signaling that the $5-15 \mathrm{~nm}$ InN film is almost completely etched from the lifted-off MLG area. After $160 \mathrm{~s}$ of Ar etching, negligible In signal is recorded for the lift-off area, whereas a strong In3d peak is still observed on nonblocked $\mathrm{Si}(100)$ area. This result shows that with $\sim 3 \mathrm{~min}$ blank Ar etch, the unwanted InN film grown under the MLG layer can be completely removed, leaving a $\sim 30 \mathrm{~nm}$ thick InN film left only on the uncovered $\mathrm{Si}(100)$ area, which corresponds to a selective III-nitride deposition featuring a selectivity of $\sim 30 \mathrm{~nm}$.

\section{2. $\mathrm{N}_{2} / \mathrm{H}_{2}$ plasma case: selective AIN deposition results}

Figures 7(a) and 7(b) show the SEM images of the open $\mathrm{Si}(100)$ and MLG-covered surface portions, respectively, after 500-cycle AlN deposition experiment, which corresponds to a similar film thickness of $\sim 50 \mathrm{~nm}$ on $\mathrm{Si}$ surface (growth-per-cycle of $\sim 1.0 \AA$ ). As opposed to InN film surface, AlN displays a more uniform morphology without distinct grain structure. The measured film thicknesses on $\mathrm{Si}(100)$ and MLG surface are very similar, which confirms the lack of any AlN nucleation inhibition characteristics of the MLG surface. Therefore, similar ultrasonication-assisted lift-off method was facilitated to remove the excess AlN on top of MLG layer. Figure 7(c) shows the SEM image of the lifted-off substrate area, which features a thinner AlN film despite the presence of the MLG blocking layer. The result is pretty similar to the $\mathrm{N}_{2}$-only plasma/InN growth case where again MLG does not function as an ideal barrier layer for AlN growth, instead enables partial shielding resulting in a reduced AlN film thickness $(5-10 \mathrm{~nm})$. Compared to the $\sim 50 \mathrm{~nm}$ film thickness on noncovered $\mathrm{Si}(100)$ area, with a successful etch process, this would correspond to an effective blocking thickness of more than $\sim 40 \mathrm{~nm}$, assuming that the unwanted AlN could be etched away completely with a dry etch process. We might attribute the unwanted AlN under-growth to similar mechanisms as we proposed for InN case. The slightly lower thickness of the under-grown AlN can be pointed out to the less harsh damaging feature of $\mathrm{N}_{2} / \mathrm{H}_{2}$ mixture when compared to $\mathrm{N}_{2}$-only plasma, which possibly cause lower surface damage in MLG and reduces the amount of under-growth. Figure 7(d) shows a highresolution SEM image of defective regions in the MLG layer, which might be partially responsible for the observed AlN under-growth besides larger cracks and side/corner peelings of the MLG flakes.

Figures 8(a) and 8(b) represent the XPS spectra collected from unblocked $\mathrm{Si}(100)$ area and MLG-protected area where MLG and the excessive AlN film on top of it is lifted-off as well as from both samples after various Ar-etch durations (20-60-120 s). The Al 2p peak on unprotected $\mathrm{Si}(100)$ area does not change after ultrasonication, while the same peak drops notably for the MLG-covered area, confirming the less AlN thickness underneath. The same Ar-etch process was applied to AlN-coated samples as well, resulting in reduced 

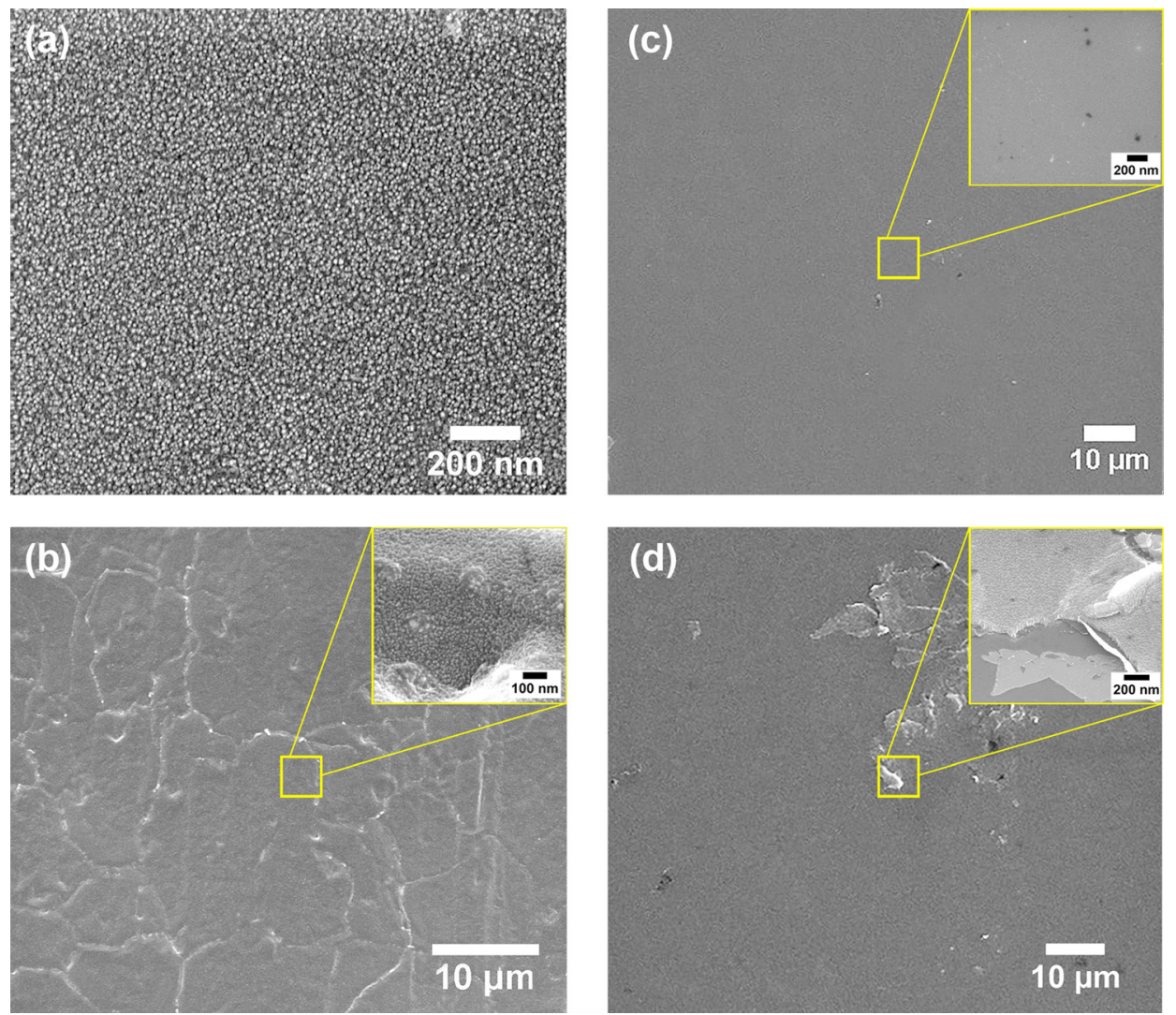

Fig. 7. (Color online) SEM images of (a) AlN film on Si(100) surface; (b) AlN film on MLG surface; (c) Si surface after lift-off process (this image belongs to the MLG blocking layer area); (d) AlN on partially defective MLG surface leading to undesired (under)growth on Si.

Al signal intensity for both $\mathrm{Si}(100)$ and lifted-off MLG regions: Al $2 \mathrm{p}$ peak completely disappears after $60 \mathrm{~s}$ Aretch, which approves the successful removal of the $5-10 \mathrm{~nm}$ AlN film from the lifted-off MLG area. After 120 s of Ar etching, the relatively strong $\mathrm{Al} 2 \mathrm{p}$ peak confirms the presence of at least $35 \mathrm{~nm}$ AlN film on the nonblocked $\mathrm{Si}(100)$ area. This result shows that with only 1 min blank Ar etch, the unwanted AlN film grown under the MLG layer can be etched away completely, leaving a $\sim 40 \mathrm{~nm}$ thick AlN film left only on the uncovered $\mathrm{Si}(100)$ area, which corresponds to a selective AlN deposition featuring a selectivity of $\sim 40 \mathrm{~nm}$.

\section{SUMMARY AND CONCLUSIONS}

We have proposed and demonstrated a methodology for low-temperature area-selective deposition of III-nitride thin films by utilizing MLG blocking layers along with subsequent lift-off and dry-etch processes. The $\mathrm{N}_{2}$-only and $\mathrm{N}_{2} / \mathrm{H}_{2}$ plasma compatibility tests for SLG and MLG surfaces showed that SLG degrades fairly quickly under both plasma chemistries resulting in disintegrated and rough surface morphology, while MLG exhibited a certain resistance and was able to preserve its continuity and integrity even after 100 cycles of plasma exposure. Based on this observation, we followed a strategy where we could use such MLG layers as blocking layers for lift-off process by using the relatively weak van der Waals bonds at the graphene-substrate interface and within MLG. To verify this methodology, plasma-assisted ALD of InN and AlN on partially MLGcovered $\operatorname{Si}(100)$ substrates was carried out. No signs of nucleation/growth inhibition on graphene surfaces was observed, resulting in an unselective $\sim 50 \mathrm{~nm}$ film deposition on both open $\mathrm{Si}(100)$ and MLG-covered surfaces. Besides, III-nitride film growth was observed in the MLG-protected areas as well, which was attributed to the presence of defects, grain boundaries, cracks, side-wall, and corner peelings formed within the MLG flakes during graphene synthesis and transfer process, allowing ample space for the precursor molecules and plasma species to diffuse for ALD growth. This unwanted $5-15 \mathrm{~nm}$ III-nitride film deposition necessitated the usage of a final etch-process step where unselective Ar-etch proved to be a promising solution. As a result, $\sim 30 \mathrm{~nm}$ thick InN and $\sim 40 \mathrm{~nm}$ AlN films were deposited selectively after graphene-assisted lift-off and blank Aretch process.

As mentioned in the title, this was a proof-of-concept study which resulted in promising achievements, yet far from being decisive and complete. To develop a precisely controlled selective deposition of III-nitrides, besides carrying out extra experiments to understand the responsible mechanisms behind plasma-graphene interactions and surface degradation, possible measures to avoid unwanted film under-growth needs to be studied as well. In addition, reliable patterning techniques such as e-beam or focused ion-beam assisted direct writing or nanoimprint lithography- 

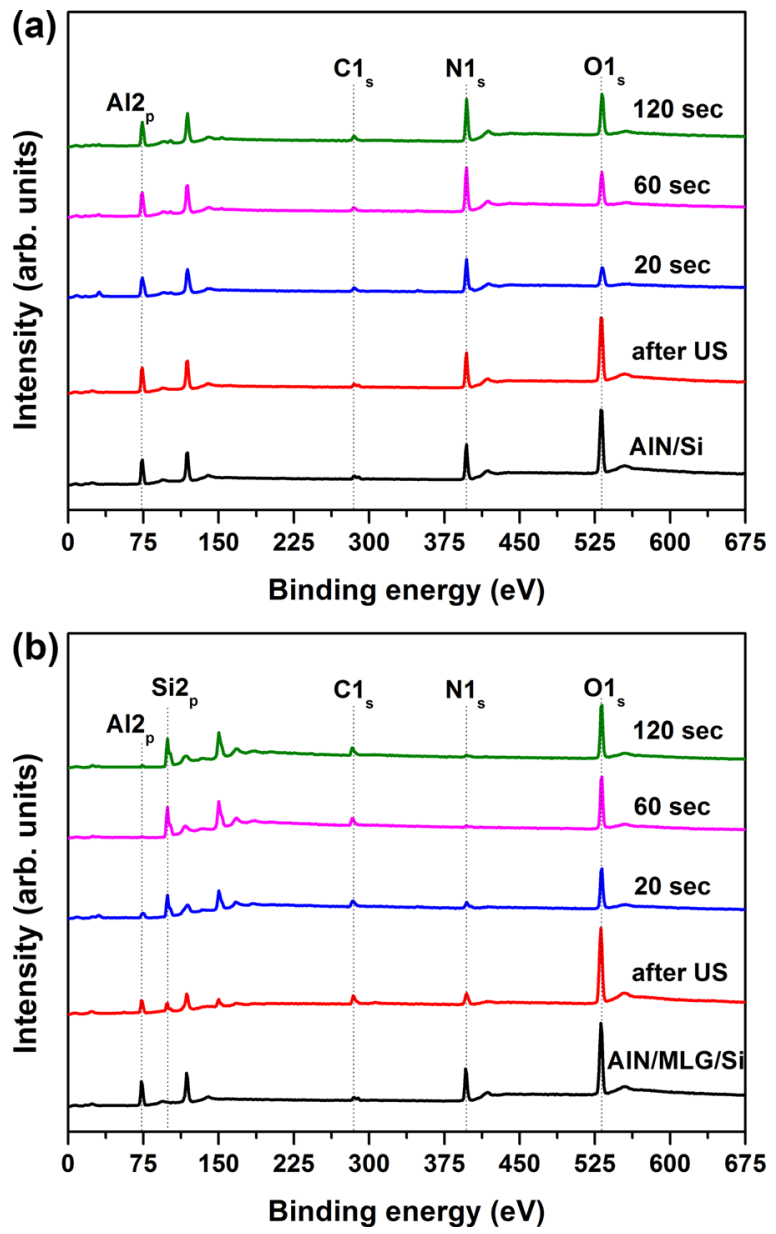

FIG. 8. (Color online) XPS survey scans of (a) AlN/Si and (b) AlN/MLG/Si surface areas before and after US and as a function of different Ar-etch times.

assisted etch processes should be experimented to demonstrate the successful selective deposition of III-nitride thin films at the micro- and nanoscale. Nevertheless, we believe that this feasibility study might spark future efforts toward the successful selective deposition of not only III-nitrides but as well other alternative nitride, oxide, and metallic films necessitating plasma usage.

\section{ACKNOWLEDGMENTS}

The authors acknowledge the National Nanotechnology Research Center (UNAM), Bilkent University for providing the materials growth and characterization facilities. P.D. acknowledges TUBITAK for financial support under the BIDEB-2216 fellowship program for international researchers. A.H. acknowledges Higher Education Commission of Pakistan (HEC) for the Human Resource Development (HRD) fellowship for MS leading to Ph.D. N.B. acknowledges support from the European Commission-Research Executive Agency (REA)-Marie Curie International Reintegration Grant (NEMSmart, Grant No. PIRG05-GA-2009-249196). N.B. also acknowledges the financial support from the Scientific and
Technological Research Council of Turkey (TUBITAK) through Grant Nos. 112M004, 112M482, and 214M015.

${ }^{1}$ C.-C. Yang, F. Baumann, P.-C. Wang, S. Y. Lee, P. Ma, J. AuBuchon, and D. Edelstein, IEEE Electron Dev. Lett. 32, 560 (2011).

${ }^{2}$ M. Mayberry, "ALD: Essential but not sufficient," in 15th International Conference on Atomic Layer Deposition (2015).

${ }^{3}$ R. L. Puurunen et al., J. Appl. Phys. 96, 4878 (2004).

${ }^{4}$ K. J. Park, J. M. Doub, T. Gougousi, and G. N. Parsons, Appl. Phys. Lett. 86, 051903 (2005).

${ }^{5}$ R. Chen, D. Porter, H. Kim, P. C. McIntyre, and S. F. Bent, Appl. Phys. Lett. 86, 191910 (2005).

${ }^{6}$ A. Sinha, D. W. Hess, and C. L. Henderson, J. Vac. Sci. Technol., B 24, 2523 (2006).

${ }^{7}$ E. Farm, M. Kemell, M. Ritala, and M. Leskela, J. Phys. Chem. C 112, 15791 (2008).

${ }^{8}$ S. M. George, Chem. Rev. 110, 111 (2010).

${ }^{9}$ C. R. Ellinger and S. F. Nelson, Chem. Mater. 26, 1514 (2014).

${ }^{10}$ F. S. M. Hashemi, C. Prasittichai, and S. F. Bent, ACS Nano 9, 8710 (2015).

${ }^{11}$ A. Haider, M. Yilmaz, P. Deminskyi, H. Eren, and N. Biyikli, RSC Adv. 6, 106109 (2016).

${ }^{12}$ S. F. Nelson, C. R. Ellinger, and D. H. Levy, ACS Appl. Mater. Interfaces 7, 2754 (2015).

${ }^{13}$ E. Farm, S. Lindroos, M. Ritala, and M. Leskela, Chem. Mater. 24, 275 (2012).

${ }^{14}$ A. Haider, P. Deminskyi, T. M. Khan, H. Eren, and N. Biyikli, J. Phys. Chem. C 120, 26393 (2016).

${ }^{15}$ W. H. Kim et al., ACS Nano 10, 4451 (2016).

${ }^{16}$ F. S. Minaye Hashemi and S. F. Bent, Adv. Mater. Interfaces 3, 1600464 (2016).

${ }^{17}$ A. J. M. Mackus, S. A. F. Dielissen, J. J. L. Mulders, and W. M. M. Kessels, Nanoscale 4, 4477 (2012).

${ }^{18}$ A. J. M. Mackus, N. F. W. Thissen, J. J. L. Mulders, P. H. F. Trompenaars, M. A. Verheijen, A. A. Bol, and W. M. M. Kessels, J. Phys. Chem. C 117, 10788 (2013).

${ }^{19}$ S. McDonnell et al., J. Phys. Chem. C 117, 20250 (2013).

${ }^{20}$ A. Mameli, Y. Kuang, M. Aghaee, C. K. Ande, B. Karasulu, M. Creatore, A. J. M. Mackus, W. M. M. Kessels, and F. Roozeboom, Chem. Mater. 29, 921 (2017).

${ }^{21}$ S. E. Atanasov, B. Kalanyan, and G. N. Parsons, J. Vac. Sci. Technol., A 34, 01 A148 (2016).

${ }^{22}$ P. C. Lemaire, M. King, and G. N. Parsons, J. Chem. Phys. 146, 052811 (2017).

${ }^{23}$ A. J. M. Mackus, A. A. Bol, and W. M. M. Kessels, Nanoscale 6, 10941 (2014).

${ }^{24}$ R. Vallat, R. Gassilloud, B. Eychenne, and C. Vallée, J. Vac. Sci. Technol., A 35, $01 \mathrm{~B} 104$ (2017).

${ }^{25}$ A. Mameli, M. J. M. Merkx, B. Karasulu, F. Roozeboom, W. M. M. Kessels, and A. J. M. Mackus, ACS Nano 11, 9303 (2017).

${ }^{26}$ E. O. Polat, O. Balci, and C. Kocabas, Sci. Rep. 4, 6484 (2014).

${ }^{27}$ E. Kovalska and C. Kocabas, Mater. Today Commun. 7, 155 (2016).

${ }^{28}$ C. Ozgit-Akgun, E. Goldenberg, A. K. Okyay, and N. Biyikli, J. Mater. Chem. C 2, 2123 (2014).

${ }^{29}$ A. Haider, S. Kizir, C. Ozgit-Akgun, E. Goldenberg, S. A. Leghari, A. K. Okyay, and N. Biyikli, J. Mater. Chem. C 3, 9620 (2015).

${ }^{30}$ A. Haider, S. Kizir, and N. Biyikli, AIP Adv. 6, 045203 (2016).

${ }^{31}$ K. Schouteden, N. Galvanetto, C. D. Wang, Z. Li, and C. Van Haesendonck, Carbon 95, 318 (2015).

${ }^{32}$ A. C. Ferrari et al., Phys. Rev. Lett. 97, 187401 (2006).

${ }^{33}$ L. M. Malard, M. A. Pimenta, G. Dresselhaus, and M. Dresselhaus, Phys. Rep. 473, 51 (2009).

${ }^{34}$ R. H. J. Vervuurt, B. Karasulu, M. A. Verheijen, W. M. M. Kessels, and A. A. Bol, Chem. Mater. 29, 2090 (2017).

${ }^{35}$ Y. Shao, S. Zhang, M. H. Engelhard, G. Li, G. Shao, Y. Wang, J. Liu, I. A. Aksay, and Y. Lin, J. Mater. Chem. 20, 7491 (2010).

${ }^{36}$ L. S. Hui, E. Whiteway, M. Hilkeb, and A. Turak, Faraday Discuss. 173, 79 (2014).

${ }^{37}$ C. Ozgit, I. Donmez, M. Alevli, and N. Biyikli, J. Vac. Sci. Technol., A 30, $01 \mathrm{~A} 124$ (2012). 